\title{
Perception Analysis and Early Warning of Home-Based Care Health Information Based on the Internet of Things
}

\author{
Yi Mao, ${ }^{1}$ Lei Zhang, ${ }^{2}$ and Xin $W u \mathbb{D}^{1,3}$ \\ ${ }^{1}$ School of Electronics and Internet of Things, Chongqing College of Electronic Engineering, Chongqing 401331, China \\ ${ }^{2}$ School of Smart Health, Chongqing College of Electronic Engineering, Chongqing 401331, China \\ ${ }^{3}$ College of Engineering and Technology, Southwest University, Chongqing 400715, China
}

Correspondence should be addressed to Xin Wu; 201330002@cqcet.edu.cn

Received 30 November 2020; Revised 30 January 2021; Accepted 4 February 2021; Published 15 February 2021

Academic Editor: Wei Wang

Copyright $(92021$ Yi Mao et al. This is an open access article distributed under the Creative Commons Attribution License, which permits unrestricted use, distribution, and reproduction in any medium, provided the original work is properly cited.

\begin{abstract}
Aiming at the problem of insufficient health monitoring of the elderly in the existing home care system, this paper designs a health information analysis and early warning system based on the Internet of Things (IoT) technology, which can monitor the physiological data of the elderly in real time. It also can be based on the elderly real-time monitoring data, physical examination data, and other types of health data, which can be used to predict diseases, so as to achieve "early detection and early treatment" of diseases. First, analyse and design the architecture and content of the home care monitoring system based on the Internet of Things. Secondly, based on the collected heart rate, blood pressure, and three-axis acceleration information of the elderly, it is analysed to determine whether the elderly are in danger of falling, and the designed system is used for early warning. Finally, this paper analyses the prediction algorithm theory of the disease prediction module in the health monitoring software of the home care system. In order to improve the accuracy of prediction, the DS evidence theory is used to optimize the traditional BP neural network (BPNN) algorithm and conduct experimental tests. The test results show that the health information analysis and early warning software of the home care system meet actual needs and achieve the expected goals.
\end{abstract}

\section{Introduction}

With the increasingly obvious trend of population aging and the increasing number of " $4+2+1$ " households, the society's requirements for elderly care services are increasing, and traditional institutional elderly care can no longer meet the increasing demand for elderly care [1]. Nowadays, home care for the elderly is mostly for the elderly living in their own homes, supplemented by community care services for the elderly. The elderly can live in their own homes and receive corresponding care from community services to meet the needs of the elderly in daily life, such as medical, health, and daily life. However, there are many disadvantages in this kind of community for the elderly at home, such as the inability to know the needs of the elderly in real time, and the elderly who have a heart attack but cannot actively send out distress signals [2-5]. Therefore, home care needs to combine the Internet of Things (IoT) technology to build a smart home system, obtain the precise needs of the elderly in real time through intelligent perception technology, and provide services for the elderly according to their needs, such as controlling smart home equipment or selecting service providers and notifying service providers to provide services.

According to data released by relevant United Nations agencies, from 2005 to 2050, the number of people over 60 years old in the world will increase from the current $8 \%$ to $16 \%$, and there will be more than 400 million people over 80 years old. Therefore, aging is a major issue facing all humanity problems. The latest statistics released by the US Census Bureau show that there are 35 million elderly citizens over the age of 65 in the United States, accounting for $12.4 \%$ of the population [6, 7]. It is estimated that, by 2030, the elderly population in the United States will sharply increase to about 70 million, and the proportion of the total population will increase to $20 \%$. That is, one out of five people 
will have grey-haired old people who depend on the social security system and their own pensions. Most Americans are unwilling to rely on their children after retirement. They generally choose to spend their old age in their own homes or live in elderly apartments and nursing homes [8]. Therefore, the smart home care system is a "life partner" they rely on very much.

In terms of health management for the elderly, many achievements have been made abroad. The Honora Company in the United States has developed an online market platform for elderly care services. Caregivers need to fill in their qualifications, skills, service types, service hours, and other pieces of relevant information on the platform when they log on to the platform for the first time [9]. The elderly also need to improve their personal information and service needs, and then the Honora platform will match the caregiver information with the needs of the elderly according to the supply-demand relationship to achieve service docking. The Etchemin research team in Spain has also developed a health management platform for the elderly. Unlike Honora, the Etchemin research team pays more attention to the emotional changes of the elderly while paying attention to the health data of the elderly [10]. When the elderly are in depression or negative emotional status at time, the system can accurately feel the emotional difficulties of the elderly, evaluate the mental state of the elderly, and provide the most suitable treatment for the elderly; Sweden's Tass Company has developed a mobile health monitoring system based on the Android system. The system uses various sensors to collect human body sign data (such as heart rate, blood pressure, and blood sugar) and displays these data on the mobile terminal in real time [11]. It can be based on physical data; the analysis results provide targeted health advice for the elderly. Since the concept of telemedicine has only appeared in our country for a few years, people are still relatively unfamiliar with it, so its development is relatively slow, and there is a big gap between it and developed countries, and there are relatively few research sites in my country's research work, and researchers are also quite lacking. Although smart elderly care has begun to develop in China, the elderly care service system on the market has not been established, especially the complete life assistance system for the semidisabled elderly. Today, when aging is becoming more and more serious, it is especially important to establish complete and economical support for the semidisabled elderly [12-14]. However, these methods are not eager for the Internet of Things, and there is no advanced evidence theory to improve the accuracy of BP network prediction.

IoTrefers to a variety of devices and technologies, such as information sensors, radio frequency identification technology, global positioning system, infrared sensors, and laser scanners, to collect any information that needs to be monitored in real time, connecting and interacting objects or processes, collecting all kinds of needed information, through all kinds of possible network access, realizing the ubiquitous connection between objects and people, realizing the intelligent perception, and identification and management of objects and processes. The emergence of the IoT provides convenience for home-based elderly health information perception analysis and early warning. This paper is based on the background of home care for the elderly, based on the IoT technology to develop the elderly health information analysis and early warning cloud platform. The construction of the platform mainly includes four aspects: intelligence, medical care integration, cloud computing, and big data analysis. Smart terminals and natural humancomputer interaction sensing equipment are used to collect the elderly's daily behaviour and health management data and effectively integrate elderly care and medical care services, a comprehensive elderly care service platform integrating "intelligent nursing, life assistance, health management, safety protection, and family care" on the high-performance cloud computing data platform. Through the big data mining method, the health information of the elderly can be analysed, and the possible diseases of the elderly can be predicted with early warning. In order to improve the accuracy of prediction, this paper uses DS evidence theory to optimize the traditional disease prediction algorithm based on BP neural network (BPNN) to improve the accuracy of disease prediction. Using $\mathrm{B} / \mathrm{S}$ architecture and Web Service technology, the main function is to perform health analysis and daily behaviour analysis on the data collected by the front end. At the same time, various dangerous behaviour warnings, health reports, health recommendations, health service information, and so on are regularly pushed for the elderly and their relatives.

The rest of this paper is organized as follows: the related theories and technologies are given in Section 2 in detail; Section 3 illustrates the health information analysis and disease prediction for the elderly at home. Simulation results, performance analysis, and conclusions are given in Section 4 and Section 5.

\section{The Structure Design of Elderly Health Monitoring System}

2.1. Elderly Health Monitoring System Based on IoT. The entire home care health monitoring system is designed to be divided into three layers, namely, the perception extension layer, the network layer, and the application service layer. The overall architecture is shown in Figure 1. The function of the perception extension layer is responsible for collecting information; it uses a variety of sensing devices and should have comprehensive perception capabilities and higher sensitivity. The network layer is the nerve of the entire system. Its main function is to transmit information. After connecting the sensing device to the wireless transmission network, the collected data can be transmitted to the upper layer. Finally, the application service layer, which is the upper platform of the whole system, is specifically responsible for providing services to users.

As can be seen from Figure 1, the system is mainly divided into perception extension layer, network connection layer, and application service layer. In perception extension layer, the sensing device that collects health data can measure the blood pressure, blood sugar, heart rate, body temperature, and so on of the human body. It has the 


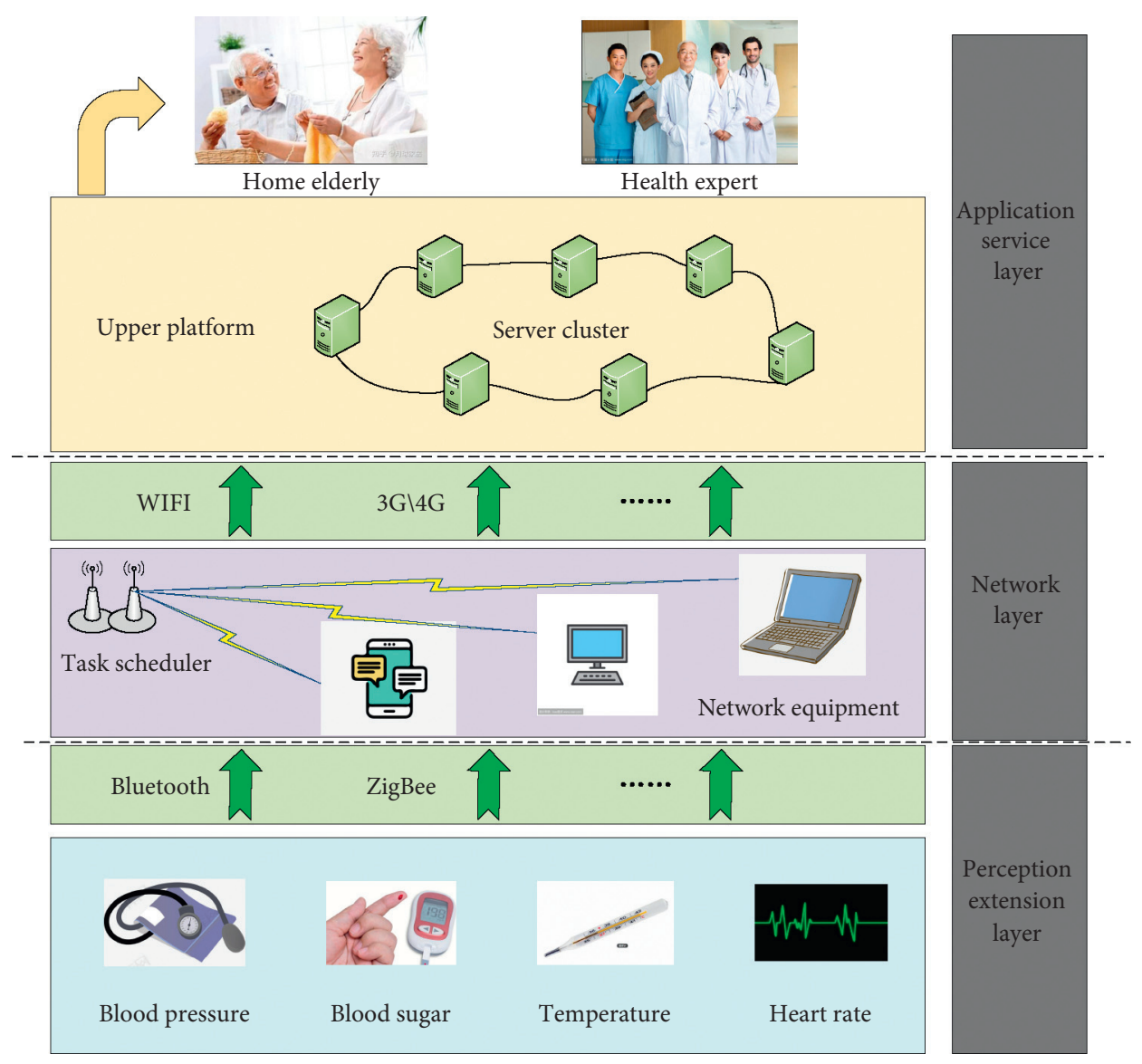

FIgURE 1: The overall architecture of the health information service system.

function of sensing and sending data, and it has low power consumption to meet long-term requirements anytime and anywhere. To collect data locally, PAN networks such as Bluetooth and ZigBee can meet its communication needs. The abovementioned digital information is transmitted to the central node deployed in the home for further processing, and after processing, the information is transmitted to the data processing server of the application service layer through WIFI, 4G, and other networks. In addition, the transmitted data may not be within the normal threshold range; then the alarm procedure should be activated in time, but sometimes it may be only occasional abnormalities, such as network abnormalities and temporary abnormal perceptions; we should not treat them taking into account that these are all within the functional scope of the data processing server. In the application service layer, we use the advanced BP network and evidence theory fusion method to predict and analyse the data, which can better improve the prediction accuracy of the system.

The application service layer also contains a web server, which can respond to users' active requests, manage users' health information through the web, provide communication channels between patients and doctors and patients and patients, and manage users' personal contact information, home address, and guardian personal information such as contact information. There is also a database server; its function is to store the historical and real-time health data of each user for web programs to access and query and can generate intermediate results to provide services for subsequent requests.

The servers can be deployed on different hosts, or they can all be deployed on one host. Although the computer has multitasking and multiprocess processing capabilities, the CPU and memory resources of a physical host are limited. When multiple servers are deployed, slow response will occur, resulting in failure to provide services to users in time, resulting in consequences of user loss and poor experience. Therefore, a program server that responds to a large number of requests can be deployed on a host. When the number of users becomes larger and larger, it is necessary to add servers to provide the processing capacity of server cluster load balancing.

\subsection{The Configuration of Home-Based Elderly Health Service} System. Architecture selection plays an important role in software development. Choosing a suitable software architecture helps ensure that the system has good performance. Common software architectures are generally divided into $\mathrm{C} / \mathrm{S}$ (client/server) and B/S (browser/server) [15]. By analysing the respective advantages of $\mathrm{C} / \mathrm{S}$ and $\mathrm{B} / \mathrm{S}$ and combining the data processing method of this software, this paper adopts a hybrid architecture solution combining $\mathrm{C} / \mathrm{S}$ and $\mathrm{B} / \mathrm{S}$ in the home care health monitoring software system, as shown in Figure 2. 


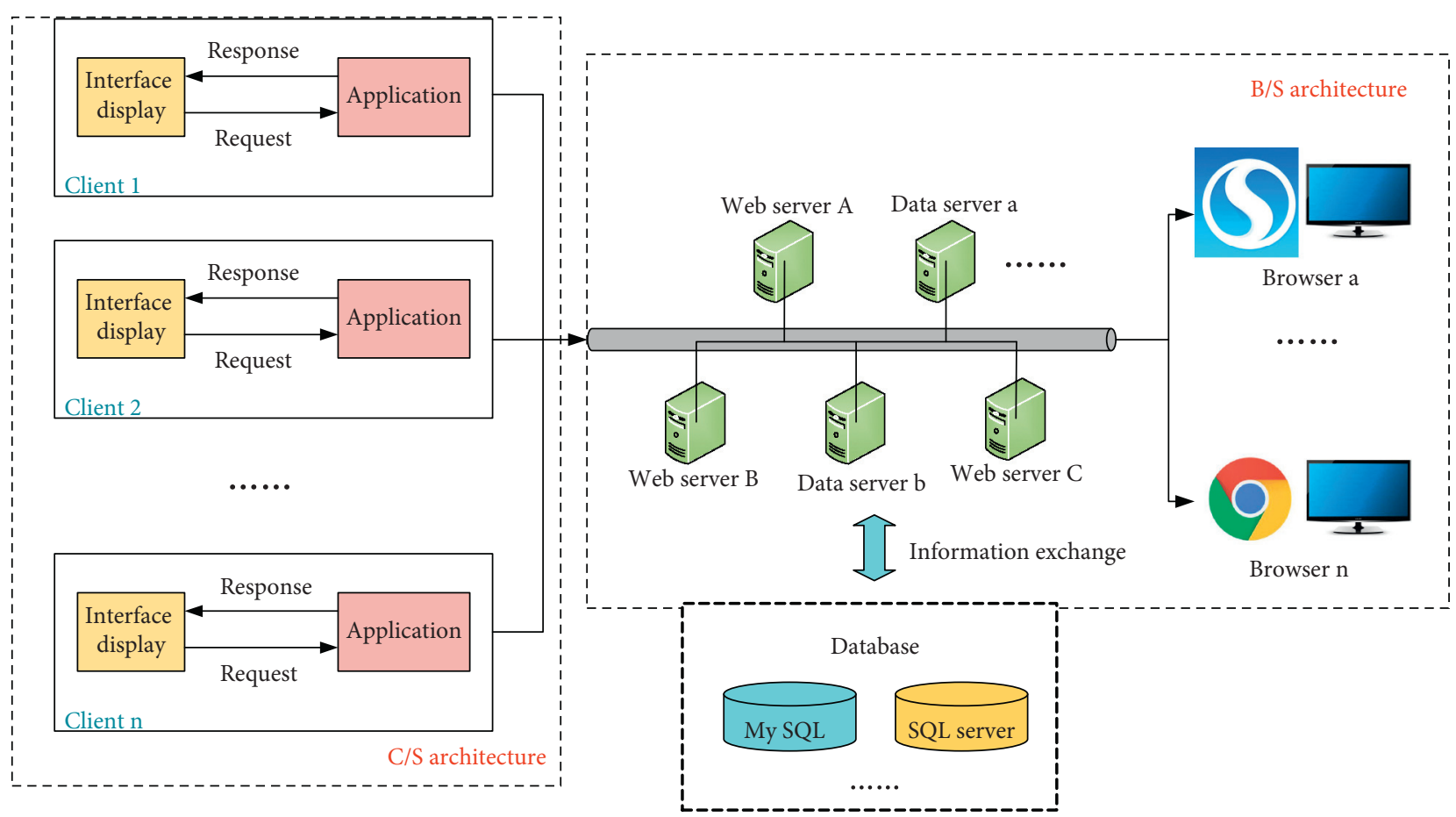

FIgURE 2: The overall architecture of the health information service system.

Taking into account the need for real-time physiological data monitoring of elderly users, the software needs to have strong transaction processing capabilities and has relatively high requirements for the timeliness and accuracy of data. Therefore, the client software for the elderly is developed using $\mathrm{C} / \mathrm{S}$ architecture with relatively high interactive capabilities and security. The system administrator and community doctors are mainly responsible for establishing health files, publishing health information, and managing user information for the elderly. Due to the wide application range of the software and the relatively low amount of information interaction, the administrator uses the $\mathrm{B} / \mathrm{S}$ model for development.

My SQL database not only has the characteristics of small size and simple installation but also can work across platforms, supports multiple languages, and is suitable for small and medium project development. In addition, My SQL also has the characteristics of free and open source, so the majority of developers love it. The health monitoring software designed in this subject is mainly for the elderly and belongs to small and medium-sized applications [16, 17]. Both SQL Server and My SQL can be used as the database of this software. However, considering the difficulty of development, compatibility, and cost, this paper chooses My SQL as the database of this software.

Since the health monitoring software used by the elderly runs on the mobile terminal, it is necessary to choose a mobile development platform. Android is an open-source system based on the Linux kernel with powerful APIs, which is very suitable for developers to develop various application software according to their needs. Considering that the IOS system development process is restricted by the development platform and the Android system has a strong user base, especially the elderly who use Android phones, the elderly mobile phone terminal of this health monitoring software chooses the Android operating system for software development. The system background adopts the currently popular Spring Boot and Mybatis integration framework. Spring Boot is spring's microservice product [18]. It integrates Spring MVC and realizes automatic configuration at the same time, avoiding the cumbersome operation when using spring framework files and saving the developer's time for configuration files. As a development framework for the data persistence layer, My Baits provides an ORM (Object Relational Mapping) mechanism to map SQL statements to java objects, simplifying the development work. Compared with Hibernate, although Mybatis requires developers to write SQL and interfaces, which increases the workload, it is precisely for this reason that Mybatis can optimize and modify SQL statements and has higher flexibility.

\section{Health Information Analysis and Disease Prediction for the Elderly at Home}

3.1. Analysis of BPNN Disease Prediction Algorithm. BPNN is the most widely used neural network. It has strong learning ability, highly nonlinear mapping ability, and largescale parallel computing ability. It is now used in image processing [16], economic forecasting [17], network classifiers [18], and other fields, which have been widely used. In the medical field, BPNN is widely used in the prediction and classification of diseases. For example, people used the BPNN to construct a schizophrenia prediction model and predict whether the schizophrenia occurred according to the selected 10 factors that affect the onset of schizophrenia. The prediction accuracy rate reached 87.5. Others used the 
BPNN to predict whether patients with cerebrovascular disease will have nosocomial infections and build a prediction model to assess the risk of nosocomial infections in patients with an accuracy rate of $89.2 \%$ [19]. In addition, the BPNN is also used in combination with other models to predict diseases. This health monitoring software builds a disease prediction model based on various physiological data of the elderly, provides the elderly and doctors with as accurate prediction results as possible, and helps the elderly prevent the occurrence of diseases in time.

The BPNN is a feedforward neural network with three or more layers. The network model includes an input layer, a hidden layer, and an output layer. There can be one or more hidden layers. In the BPNN structure, there is no feedback loop between layers, and there is no interconnection between nodes in the same layer [20]. Some scholars have theoretically proved that when the number of hidden layer nodes can be adjusted freely as needed, a three-layer neural network (i.e., only one hidden layer) can approach any continuous nonlinear mapping with any accuracy. Figure 3 shows a typical three-layer BPNN structure and its algorithm flow.

Constructing the BPNN structure is the first step in the realization of the BPNN algorithm. Take the three-layer BPNN in Figure 3 as an example. The network has $n$ inputs and $m$ outputs, and the hidden layer has $l$ neurons. The output of the $j$ th neuron in the hidden layer is $h_{j}, y_{k}$, and $d_{k}$ are the predicted output and expected output of the $k$-th neuron in the output layer, respectively. After determining the number of nodes in each layer of the system, it is also necessary to initialize the weight and threshold of the system (threshold is also called bias), where $w_{i j}$ represents the connection weight between the input layer and the hidden layer and $w_{j k}$ is the hidden layer. The connection weights between the output layers, $a_{j}$ and $b_{k}$, are the thresholds of the corresponding neurons in the hidden layer and the output layer. The learning rate is $\eta$, the calculation accuracy value is $\varepsilon_{0}$, the maximum number of learning is $N_{0}$, and the neuron activation function is $f(x)$, where $f(x)$ takes the Sigmoid function, in the form:

$$
f(x)=\frac{1}{1+e^{-x}} .
$$

After constructing the structure of the neural network and initializing the network, it enters the training phase of the neural network, which is also called the learning process of the BPNN [21]. Use the gradient descent method to update the weights and thresholds of the hidden layer to the output layer and the input layer to the hidden layer by layer and finally get the weight update formula shown in formula (2) and the threshold update formula shown in formula (3):

$$
\begin{aligned}
& \left\{\begin{array}{l}
w_{i j}=w_{i j}+\eta h_{j}\left(1-h_{j}\right) x_{i} \sum_{k=1}^{m} w_{j k} e_{k}, \\
w_{j k}=w_{j k}+\eta h_{j} e_{k} .
\end{array}\right. \\
& \left\{\begin{array}{l}
a_{j}=a_{j}+\eta h_{j}\left(1-h_{j}\right) \sum_{k=1}^{m} w_{j k} e_{k}, \\
b_{k}=b_{k}+\eta e_{k} .
\end{array}\right.
\end{aligned}
$$

The essence of BPNN training is a process of continuously adjusting weights and thresholds. The condition for stopping training is that the network error drops to a set minimum value or reaches a set number of training steps. After the training is successful, save the weight and threshold to obtain the network and then input the prediction sample into the trained network to obtain the prediction result.

\subsection{Analysis of Elderly Disease Prediction Algorithm Based on} DSBP Neural Network. Because the BPNN is difficult to determine the network structure when making predictions, it is easy to fall into local minima and other shortcomings. In this section, the DS evidence theory is used to optimize the BPNN algorithm, and a new disease prediction model is obtained, which is collectively referred to as the DSBP model below. The model uses the DS combination rule to fuse multiple sets of data obtained by the BPNN into one set of data, thereby improving the prediction accuracy and accuracy of the BPNN.

DS evidence theory can meet weaker conditions than Bayesian when dealing with uncertainty problems, and it has obvious advantages and is currently widely used in information fusion, decision analysis, pattern recognition, and other fields. Suppose that the universe $U=\left\{x_{1}, x_{2}, \ldots, x_{n}\right\}$ is a set of all propositions in a hypothetical space, and the elements in $U$ are mutually exclusive; then $U$ is called the recognition frame. $2^{U}$ is the power set composed of all subsets of the identification frame $U$. If the function $\mathrm{m}$, $2^{U}->[0,1]$, satisfies formulas (4) and (5), then $m(A)$ is called the basic probability assignment (BPA) of $A$, which represents the degree of trust in hypothesis $A$.

$$
\begin{aligned}
m(\varnothing) & =0, \\
\sum_{A \subseteq U} m(A) & =1 .
\end{aligned}
$$

Formula (4) indicates that no support is generated for the empty set, and formula (5) indicates that the sum of the trust values of all hypotheses must be one. When $m(A)>0$, call $A$ the focal element of $m$. The trust function bel and likelihood function $\mathrm{pl}$ of the proposition are defined as

$$
\begin{aligned}
\operatorname{bel}(A) & =\sum_{B \mid B \subseteq A} m(B), \\
\operatorname{pl}(A) & =1-\operatorname{bel}(\bar{A})=\sum_{B \mid B \cap A \neq \varnothing} m(B) .
\end{aligned}
$$

According to formula (6) and formula (7), combined with the confidence interval graph in Figure 4, it can be seen that the trust function $\operatorname{bel}(A)$ of $A$ is the sum of the BPA of each subset $B$ in $A$, which represents the assumption that $A$ is really trust level. The likelihood function of $A, \operatorname{pl}(A)$, is the sum of the BPA of all hypotheses that intersect with $A$ and is not empty, indicating that the degree of doubt that $A$ is true. For a certain hypothesis $A$ in a recognition framework, its uncertainty interval is the trust function and the likelihood. The closed interval $[\operatorname{bel}(A), \operatorname{pl}(A)]$ formed by the function represents the uncertainty of hypothesis $A$. Then the 


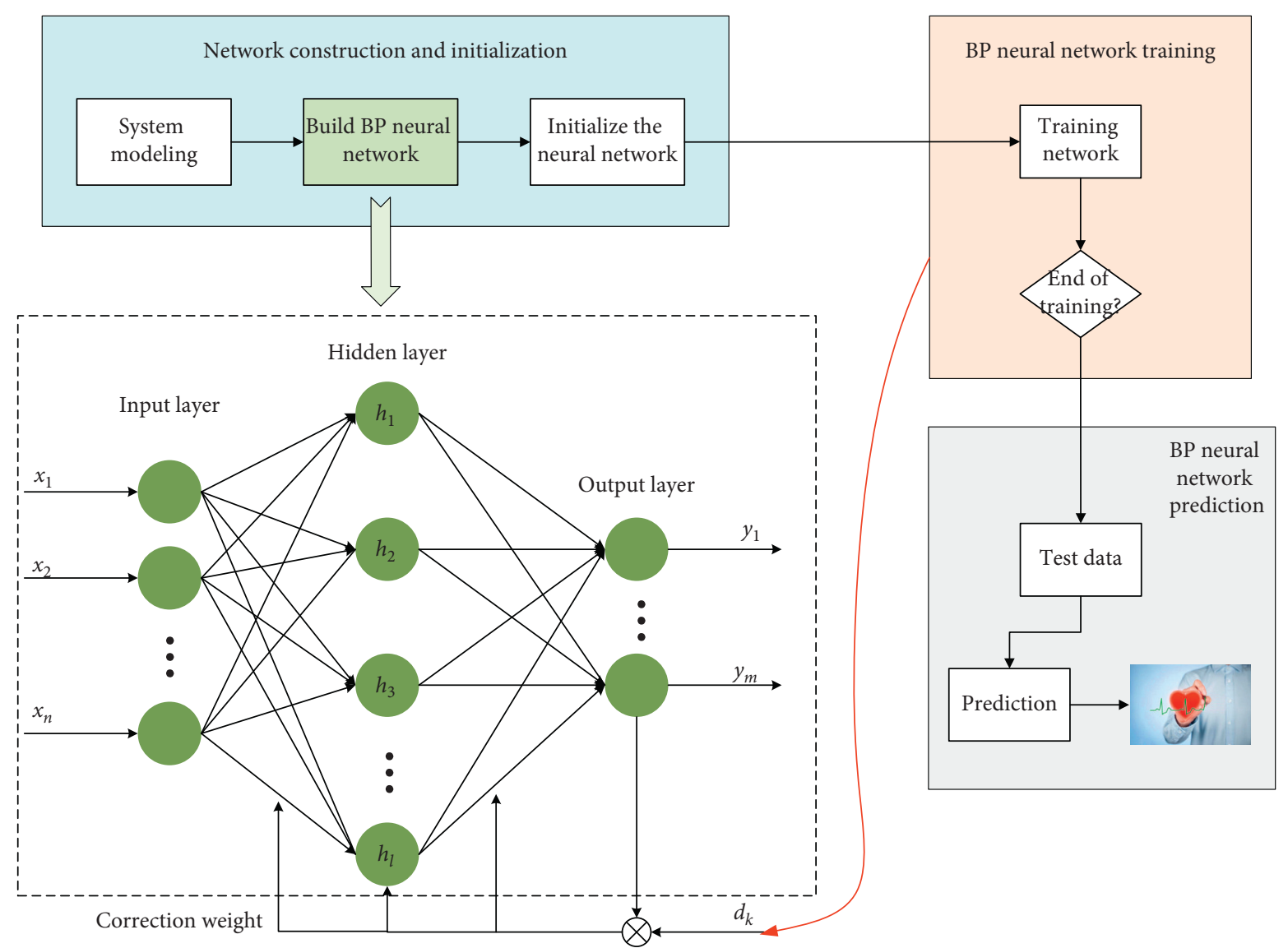

FIGURE 3: BP neural network (BPNN) algorithm structure and process.

combined evidence obtained after combining the two independent pieces of evidence, $m 1$ and $m 2$, is

$$
\begin{aligned}
m_{12}(C) & = \begin{cases}(1-K)^{-1} \sum_{A \cap B=C} m_{1}(A) m_{2}(B), & \forall C \subset U \text { and } C \neq \varnothing, \\
0, & C \neq \varnothing,\end{cases} \\
K & =\sum_{A \cap B=\varnothing} m_{1}(A) m_{2}(B)<1 .
\end{aligned}
$$

The DS combination formula satisfies the commutative law and associative law and can combine several pieces of independent evidence to obtain a more accurate BPA. In the formula, $K$ is called the conflict coefficient, and its magnitude is positively correlated with the conflict between pieces of evidence. The greater the value of $K$, the greater the conflict.

In the DSBP prediction algorithm, the BPNN is still the main part of the algorithm, and its purpose is to obtain multiple sets of probability vectors for use in DS synthesis. The DS evidence theory only needs to fuse the output results of the BPNN according to the combination rules, fully considers the potential relationship between the pieces of evidence, and converts multiple sets of probability vectors into a set of probability vectors to obtain results that are more accurate and improve prediction accuracy. Figure 4 shows the DSBP prediction model framework. The entire prediction process includes the training process of the $\mathrm{BPNN}$, the prediction process of the BPNN, and the process of using the DS evidence theory to fuse data.

The whole prediction model includes three steps, the training of traditional BPNN, the prediction process of BPNN, and the use of DS evidence theory to fuse data. The multiple BPNN prediction results obtained above are normalized and converted into the basic probability distribution that meets the DS evidence theory conditions. Then use the DS combination formula to fuse the data to get the unique probability distribution, and finally get the final prediction result according to the decision rule. 


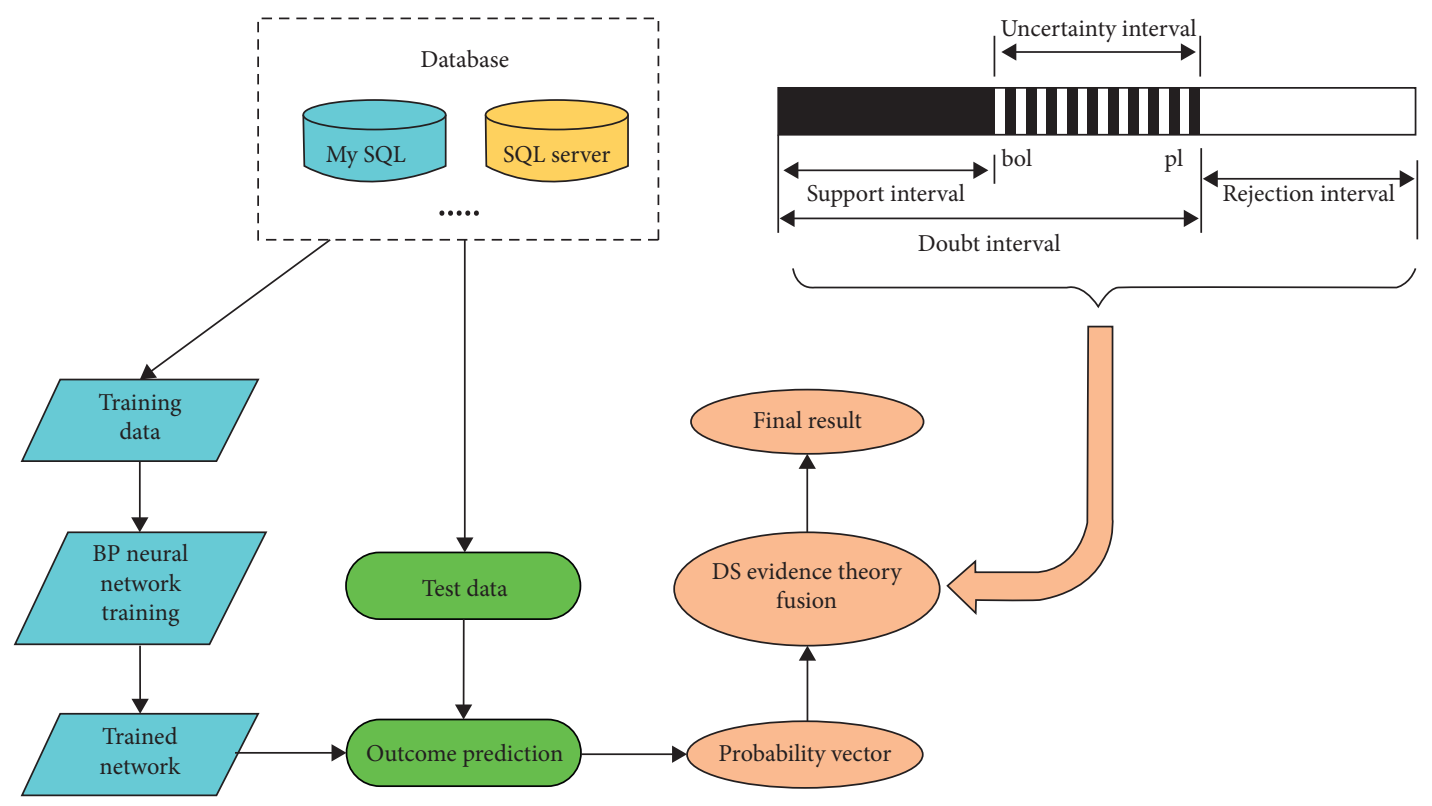

FIGURE 4: DSBP prediction model framework.

\section{Simulation Results and Performance Analysis}

4.1. Analysis of the Early Warning Results of Falls for the Elderly. The three-axis acceleration sensor and gyroscope are used to measure relevant characteristic values to determine whether a drop has occurred. The axial acceleration sensor can sense the acceleration of the object in the $X, Y$, and $Z$ directions in space, and the gyroscope can measure the physical angular movement very well. In the system, we use modular three-axis accelerometers and gyroscopes to collect body motion information, then use feature value discrimination algorithms to analyse whether the human body falls, and use IoT technology to provide early warning to family members and health experts. In order to provide early warning of the most common fall risks in the home life of the elderly, the experimental collection results of the three-axis accelerometer were analysed. The acceleration of the elderly in all directions collected by the three-axis accelerometer is shown in Figure 5.

Figure 5 shows the experimental test results of the threeaxis acceleration sensor when the elderly are walking normally at home and are in danger of falling. The image on the left shows the experimental result data normally. It can be seen that when the elderly walk normally, only the acceleration change in the $y$ direction exists, and the acceleration value in other directions is zero. The picture on the right is the experimental result data when the elderly are in danger of falling. It can be seen that when the elderly is in danger of falling, the acceleration of the $z$-axis is obviously increased. At the same time, 0.1 also increases the acceleration of the $x$ axis. On the contrary, the acceleration of $y$-axis has dropped significantly. It shows that the old man stopped and fell sideways, consistent with the actual situation. When the system detects the fall signal of the elderly, it will provide real-time warning through the IoT system described in Section 2 and notify the family and health experts.
4.2. Stability Analysis of BPNN for Predicting Diseases of the Elderly at Home. In order to verify the stability of the proposed algorithm, first initialize the commonly used parameters and set the learning rate (generally selected between 0.01 and 0.8); the maximum number of training times is 200 , the mean square error is set to 0.01 , and the hidden layer activation function uses the Sigmoid function. Then the network structure is determined. This experiment uses a three-layer BPNN structure to predict heart disease. For the number of input nodes, since there are 13 factors related to heart disease, 13 nodes are selected, and the number of output nodes is the number of heart disease types. According to the original data, the number of nodes in the output layer of the model is 5 . Before training the BPNN, it is necessary to initialize the connection weights and thresholds between neurons in each layer. In general, the connection weight and threshold are random numbers. In this experiment, a random number between $[-1,1]$ is used as the initial value of the connection weight and threshold. Perform neural network training until all samples are trained or the mean square error reaches $10^{-7}$; save the weights and thresholds to obtain the network for subsequent experiments. The error curve obtained by simulation is shown in Figure 6. The thermal map of the adaptive function test results is shown in Figure 7.

It can be seen from Figure 7 that the function has a large number of local minima distributed in the domain, and the global minima are at $(0,0)$. A large number of local minima increase the difficulty of finding the global minimum for the BPNN algorithm. It can be seen from Figure 6 that, after 10 iterations, the mean square error reaches $10^{-7}$, and the training ends.

4.3. Analysis of Experimental Test Results of DSBP Prediction Algorithm. Before verifying the disease prediction algorithm in this paper, it is necessary to select disease data. This paper 

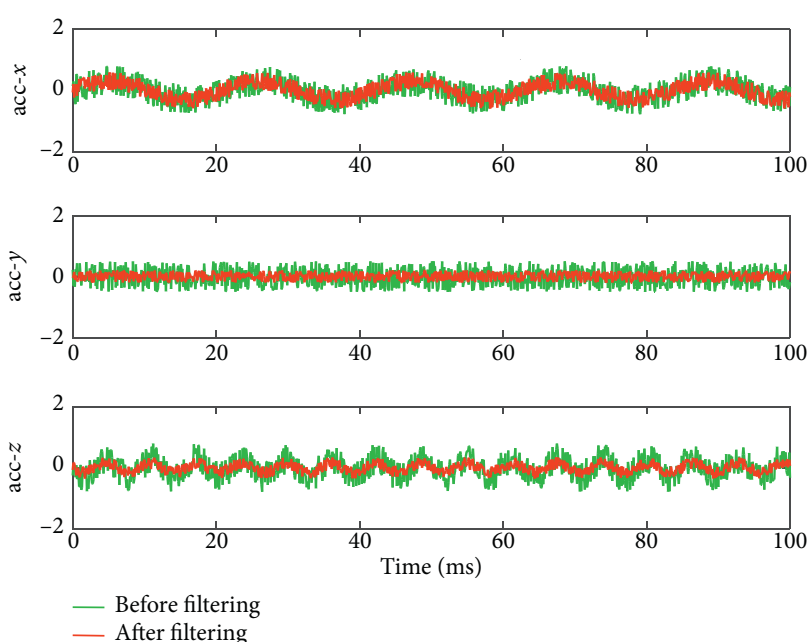

FIGURE 5: Three-axis acceleration collection results for the elderly at home.

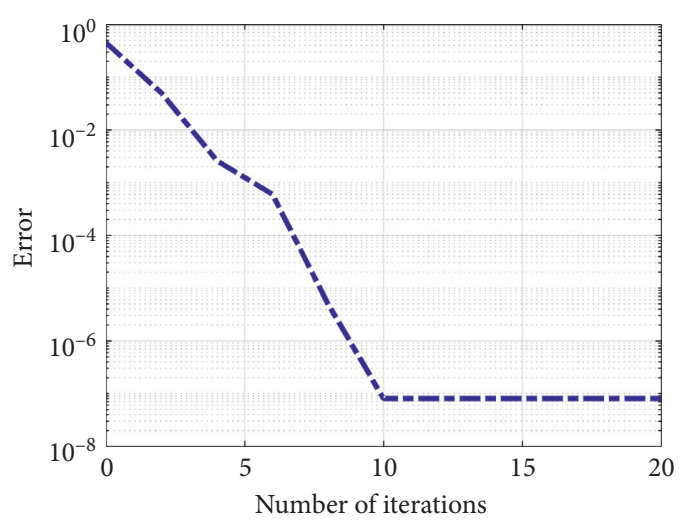

FIGURE 6: BPNN training error curve.

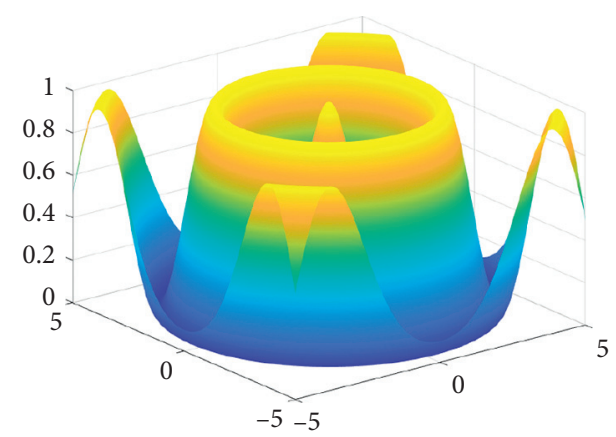

FIgURE 7: BPNN Schaffer function test results.

selects the heart disease data set on the official website of UCI standard data set for verification. The data includes 14 attributes such as age, gender, and type of chest pain. In the original data of some heart disease data sets, num represents the type of heart disease ( 0 means no disease; $1-4$ mean 4 types of heart disease).
It is found that most of the age attributes of the data are over 50 years old, so this data set is selected to build a predictive model to achieve a certain degree of reliability in predicting heart disease in the elderly. In this experiment, the 600-prediction data in the data set are divided into 200 groups according to the principle of similar data characteristics and the same result, with 3 data in each group. Therefore, predicting and verifying these 600 pieces of data is equivalent to experimenting with 200 subjects, each of which contains 3 pieces of data. The 200 sets of test samples were tested according to the type of heart disease, with 40 subjects in each type. Figure 8 counts the recognition rate of the BPNN, also called the prediction accuracy rate, that is, the ratio of the number of correct predictions to all the prediction data of this type. Among them, BP1 represents the result obtained through BPNN using the first data for the sample objects, and BP2 and BP3 are similar. Three different experiments are used to verify the role of evidence theory and eliminate the uncertainty caused by abnormal data.

This paper will compare the BPNN prediction algorithm with the DSBP algorithm in terms of prediction accuracy and robustness, to judge the pros and cons of the algorithm. The 200 sets of prediction data are all passed through the neural network to obtain the preliminary prediction results, then the final probability distribution after the fusion is obtained through the DS evidence theory, the type of heart disease is determined, and the prediction accuracy is calculated. In addition, compare the accuracy rate obtained through the DSBP algorithm with the accuracy rate obtained through the BPNN for a single piece of data of each object.

It can be seen from Figure 9 that the accuracy of the prediction results obtained through the neural network based on only a single piece of data is significantly lower than that obtained by fusing the BPNN prediction results of multiple pieces of data using DS evidence theory. After the network algorithm is improved, the DSBP algorithm has a good improvement in the prediction accuracy.

In order to verify the robustness of the algorithm, this paper sets a threshold in both prediction models, stipulating that the prediction result is valid only when the prediction result is greater than or equal. Increase the threshold in turn, and verify the robustness of the algorithm according to the change in the accuracy of the algorithm's prediction. The robustness of the algorithm also reflects the accuracy of the algorithm. That is, the degree of confirmation of a certain type of disease is greater. The trend of BP and DSBP prediction accuracy with the threshold is shown in Figure 9.

From Figure 10, it can be seen that as the threshold set by the system increases, it indicates that the judgment requirements for disease prediction results are becoming more and more stringent, and the prediction accuracy of the two algorithms is showing a downward trend. Among them, for the BPNN prediction model, when the threshold is set less than 0.85 , the prediction accuracy is 


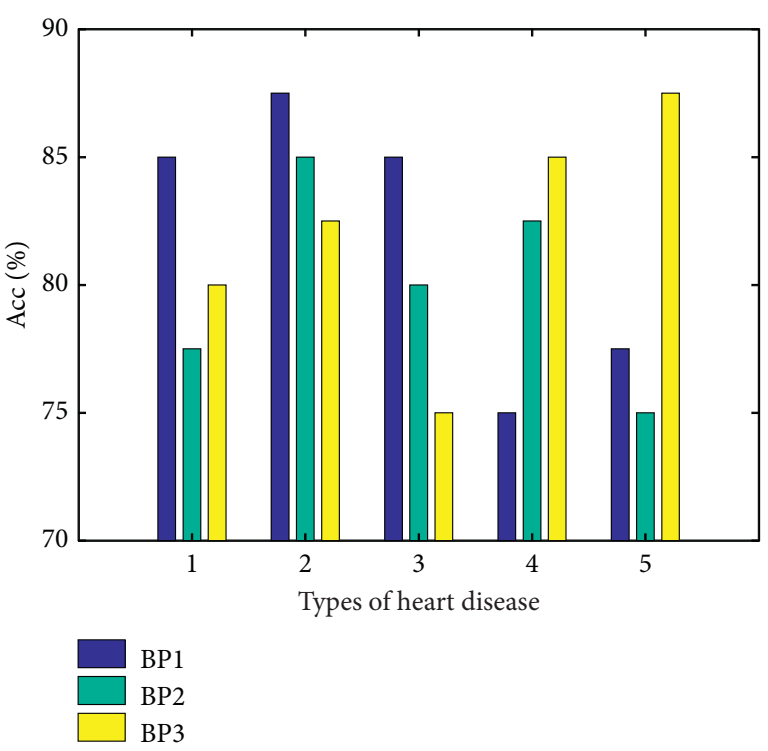

Figure 8: BPNN heart disease recognition rate.

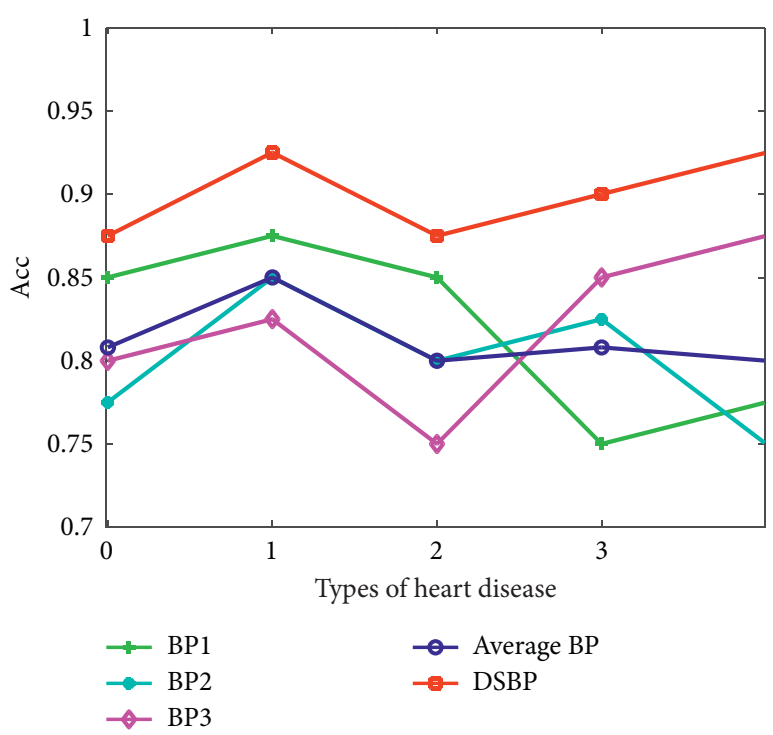

FIgURE 9: Comparison of prediction accuracy between BP algorithm and DSBP algorithm.

higher. But when the threshold is greater than 0.85 , the downward trend of the prediction accuracy rate becomes faster and the accuracy rate decreases, indicating that the stability of the BPNN is not good and the accuracy is not high. When the threshold is increased to 0.97, the BPNN model only has a prediction accuracy of 0.127 , and the algorithm is not robust. As for the DSBP model, with the increase of the threshold, although the prediction accuracy rate shows a certain downward trend, its prediction accuracy rate has been maintained at a high level. Even when the threshold is as high as 0.97 , the DSBP

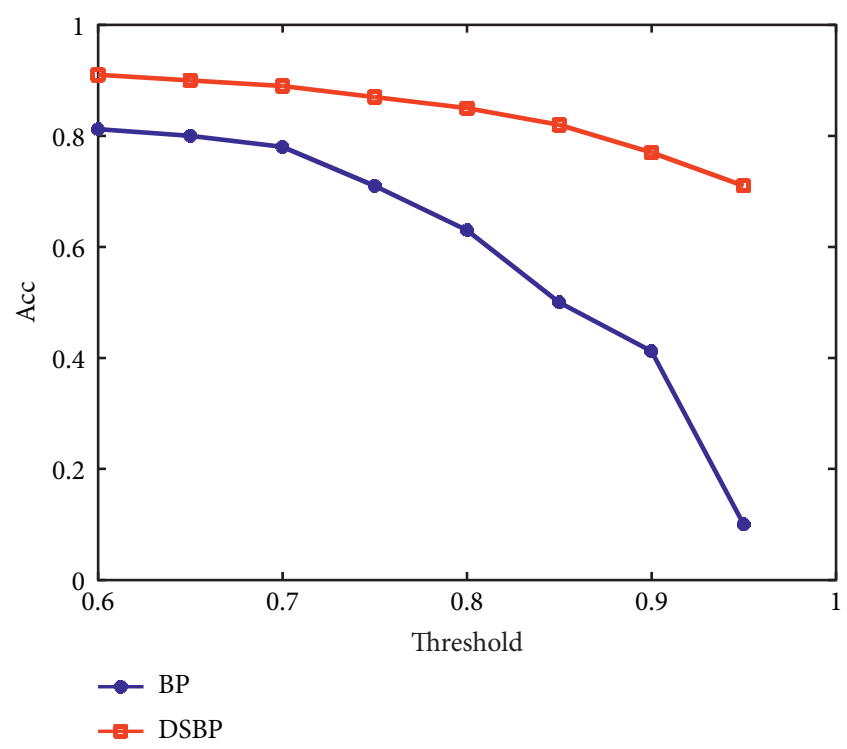

FIGURE 10: The trend graph BP and DSBP prediction accuracy rate with threshold change.

model has a prediction accuracy of 0.825 , and its robustness is significantly higher than the BPNN prediction model.

\section{Conclusion}

In order to prevent accidents when the elderly care at home, this paper studies the health information analysis and early warning system for the elderly based on the IoT technology. First, based on the research on the current home care system, a three-tier home care monitoring system based on the IoT technology is proposed, and the overall structure of the system is designed. The system not only completes the monitoring of real-time health data of the elderly but also judges the risk of falling for the elderly based on the collected data and provides real-time early warning through the IoT technology. At the same time, disease prediction is carried out based on the historical health data of the elderly, and the "early detection and early treatment" of the disease is also important for health monitoring. Therefore, this paper selects and analyses the disease prediction algorithm and compares the traditional $\mathrm{BP}$ the neural network algorithm has improved to form an improved DSBP disease prediction algorithm. This method uses DS evidence theory to process the results of the BPNN algorithm to obtain more accurate prediction results.

\section{Data Availability}

The data used to support the findings of this study are available from the corresponding author upon request. 


\section{Conflicts of Interest}

The authors declare that they have no conflicts of interest.

\section{Acknowledgments}

This work was supported by 2019 Youth Project of Science and Technology Research Program of Chongqing Education Commission of China "Research and Application of Smart Home Care Pension Service Based on Big Data" (no. KJQN201903105); Chongqing Science and Technology Commission Projects (no. cstc2018jscx-msybX0163); and 2018 Youth Project of Science and Technology Research Program of Chongqing Education Commission of China based on ZigBee Wireless Sensor Network of Intelligent Cold Storage Integrated Monitoring System (no. KJQN201803101).

\section{References}

[1] G. Mei, N. Xu, J. Qin et al., "A survey of internet of things (IoT) for geohazard prevention: applications, technologies, and challenges," IEEE Internet of Things Journal, vol. 7, no. 5, pp. 4371-4386, 2019.

[2] M. Usak, M. Kubiatko, M. S. Shabbir, O. Viktorovna Dudnik, K. Jermsittiparsert, and L. Rajabion, "Health care service delivery based on the Internet of things: a systematic and comprehensive study," International Journal of Communication Systems, vol. 33, no. 2, p. e4179, 2020.

[3] H. Fu, M. Wang, P. Li et al., "Tracing knowledge development trajectories of the internet of things domain: a main path analysis," IEEE Transactions on Industrial Informatics, vol. 15, no. 12, pp. 6531-6540, 2019.

[4] X. Zhang, S. Liu, X. Chen, L. Wang, B. Gao, and Q. Zhu, "Health information privacy concerns, antecedents, and information disclosure intention in online health communities," Information \& Management, vol. 55, no. 4, pp. 482-493, 2018.

[5] J. K. Jensen, R. Skår, and B. Tveit, "Introducing the national early warning Score - a qualitative study of hospital nurses' perceptions and reactions," Nursing Open, vol. 6, no. 3, pp. 1067-1075, 2019.

[6] J. C. Ginestra, H. M. Giannini, W. D. Schweickert et al., "Clinician perception of a machine learning-based early warning system designed to predict severe sepsis and septic shock* ," Critical Care Medicine, vol. 47, no. 11, pp. 1477-1484, 2019.

[7] C. S. Parshuram, K. Dryden-Palmer, C. Farrell et al., "Effect of a pediatric early warning system on all-cause mortality in hospitalized pediatric patients," Jama, vol. 319, no. 10, pp. 1002-1012, 2018.

[8] I. Azimi, A. M. Rahmani, P. Liljeberg, and H. Tenhunen, "Internet of things for remote elderly monitoring: a study from user-centered perspective," Journal of Ambient Intelligence and Humanized Computing, vol. 8, no. 2, pp. 273-289, 2017.

[9] Y. Liu, L. Zhang, Y. Yang et al., "A novel cloud-based framework for the elderly healthcare services using digital twin," IEEE Access, vol. 7, pp. 49088-49101, 2019.

[10] S. Zeadally and O. Bello, "Harnessing the power of internet of things based connectivity to improve healthcare," Internet of Things, vol. 4, no. 2, Article ID 100074, 2019.
[11] S. Kang, H. Baek, E. Jung, H. Hwang, and S. Yoo, "Survey on the demand for adoption of internet of things (IoT)-based services in hospitals: investigation of nurses' perception in a tertiary university hospital," Applied Nursing Research, vol. 47, pp. 18-23, 2019.

[12] D. He, S. Chan, and M. Guizani, "Security in the internet of things supported by mobile edge computing," IEEE Communications Magazine, vol. 56, no. 8, pp. 56-61, 2018.

[13] M. M. Dhanvijay and S. C. Patil, "Internet of things: a survey of enabling technologies in healthcare and its applications," Computer Networks, vol. 153, pp. 113-131, 2019.

[14] S. X. Lin, H. Z. Liu, S. J. Chen et al., "Optimization and implementation of zigbee wireless location algorithm," Revest de la Faulted de Ingenerate, vol. 32, no. 5, pp. 396-404, 2017.

[15] L. M. Dang, M. J. Piran, D. Han, K. Min, and H. Moon, "A survey on internet of things and cloud computing for healthcare," Electronics, vol. 8, no. 7, p. 768, 2019.

[16] P. Panchatcharam and S. Vivekanandan, "Internet of things (IOT) in healthcare - smart health and surveillance, architectures, security analysis and data transfer," International Journal of Software Innovation, vol. 7, no. 2, pp. 21-40, 2019.

[17] I. de la Torre Díez, S. G. Alonso, S. Hamrioui, E. M. Cruz, L. M. Nozaleda, and M. A. Franco, "IoT-based services and applications for mental health in the literature," Journal of Medical Systems, vol. 43, no. 1, p. 11, 2019.

[18] S. El Kafhali and K. Salah, "Performance modelling and analysis of Internet of Things enabled healthcare monitoring systems," IET Networks, vol. 8, no. 1, pp. 48-58, 2018.

[19] B. Wang, W. Kong, H. Guan, and N. N. Xiong, "Air quality forecasting based on gated recurrent long short term memory model in Internet of Things," IEEE Access, vol. 7, pp. 69524-69534, 2019.

[20] M. M. Baig, S. Afifi, H. GholamHosseini, and F. Mirza, "A systematic review of wearable sensors and IoT-based monitoring applications for older adults - a focus on ageing population and independent living," Journal of Medical Systems, vol. 43, no. 8, p. 233, 2019.

[21] P. P. Ray, D. Dash, and D. De, "Edge computing for internet of things: a survey, e-healthcare case study and future direction," Journal of Network and Computer Applications, vol. 140, pp. 1-22, 2019. 